\title{
A NEW CONCEPT OF HUMIDIFICATION
}

\author{
N. N. URBANOWiCZ, C.R.I.T.T., F. O. BOND, C.R.X.T.T., \\ D. A. Pelton, M.D., F.R.C.P.(c), AND A. W. ConN, M.D., F.R.C.P. (c) ${ }^{*}$
}

AT THE PRESENT TIME there are four means of producing humidification which are commonly used in the medical application of humidity therapy. They are as follows:

A. The diffuser-head type.

B. The centrifugal disc.

C. An open throat venturi.

D. The ultrasonic generator.

The various means of producing humidity are discussed in the following paragraphs.

A. A diffuser-head (Fig. I) is a device in which a source of motive power in the form of compressed gas is connected to the humidifier by a standard threaded connection. The flow of gas is introduced into the humidifier by means of a flowmeter which is connected to the humidifier at point $A$. The gas transverses the

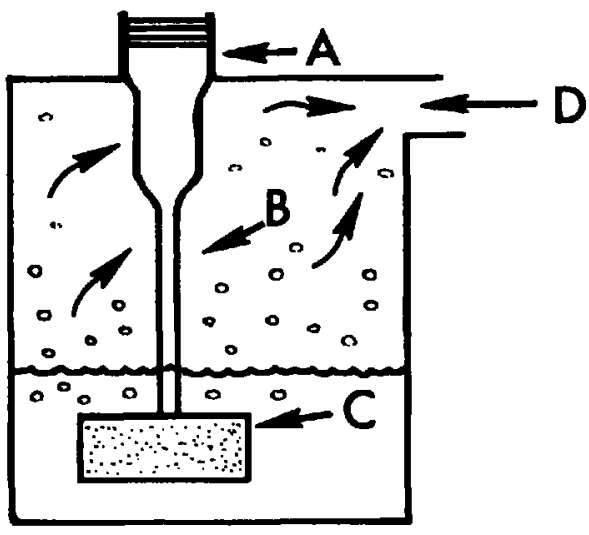

Figure 1

connecting tube $B$, into the porous stone $C$, and escapes into the water in the form of gas bubbles. Because the bubbles contain gas in a dry state, water vapour is attracted into the gas. These humidified gas particles pass from the reservoir jar through the exit port $D$, because the pressure within the jar is greater than the ambient pressure. Most of these particles are sub-micronic as they exit through the port and therefore they are not observed. However, factors such as relative humidity and temperature may affect the particle size to a degree that they then become visible.

The diffuser-head type is most aptly applied to nasal catheter therapy. Within the usual range of flow of oxygen (2-6 L./min.) adequate humidification is pro-

-Department of Anaesthesia and Department of Inhalation Therapy, Hospital for Sick Children, Toronto. 
duced. However, this device produces a small volume of humidity and will not produce adequate humidity when it is used with incubators, tents, and masks.

B. The centrifugal disc (Fig. 2) is a device in which an electric motor drives a rotating circular disc $A$, which in turn creates a negative pressure within the pick up feed tube $B$. This negative pressure draws water into the feed tube. Due to the action of the rotating disc, the water particles bombard the container producing a wide spectrum of particle sizes. The air-borne particles are then carried to the atmosphere by means of tube $C$.

This device produces large volumes of humidity, but it would appear that humidification of the lower airway is inadequate.

C. The open-throat venturi is a device in which the force of a flowing gas is used to break up a liquid into smaller particles. Figures 3 and 4 portray the

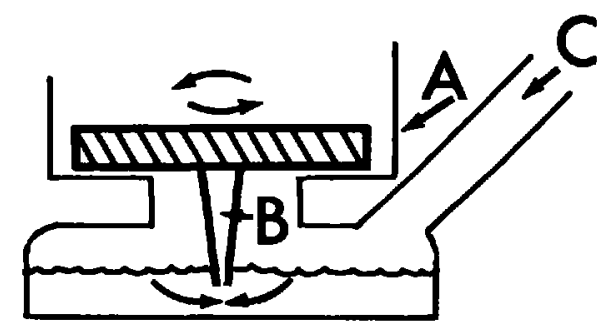

Figure 2

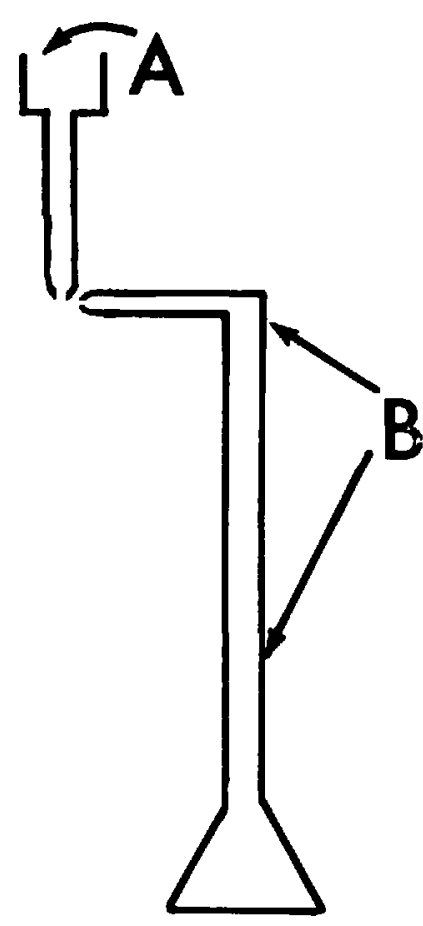

Figuhe 3

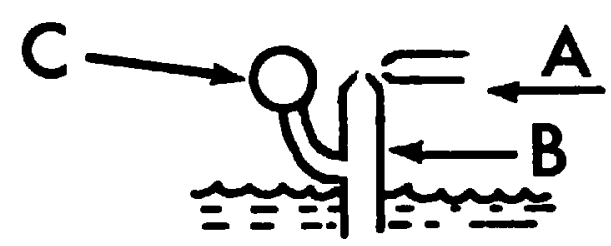

Figure 4 
utilization of the venturi principle in the production of humidity. In both figures, two tubes are set at right angles to each other and gas is forced through tube $A$. This produces a negative pressure throughout the entire length of tube $B$. Water particles are drawn up from the container into tube $B$ and exit through the orifice adjacent to tube $A$. The liquid is broken into smaller particles of varying size by the pressurized gas which exits from tube $A$. Figure 4 in addition, has a baffle $C$, which produces a further breakdown of the water particles.

This method produces a fair control over particle size and can be applied to various types of apparatus. Both large and small types of nebulizers utilizing this principle have as their main disadvantage a pronounced tendency to clog. Further disadvantages are that some medicants tend to come out of solution and some medicants cannot be nebulized owing to their high viscosity.

D. A recent development, the ultrasonic nebulizer (Fig. 5), is a device in which an electrical source of energy $A$ is converted into mechanical energy in a transducer $B$. The mechanical energy (high frequency) causes the crystal $C$ to vibrate at the frequency produced. Water is allowed to come in contact with the vibrating crystal and is broken into smaller particles. The frequency will determine the particle size.

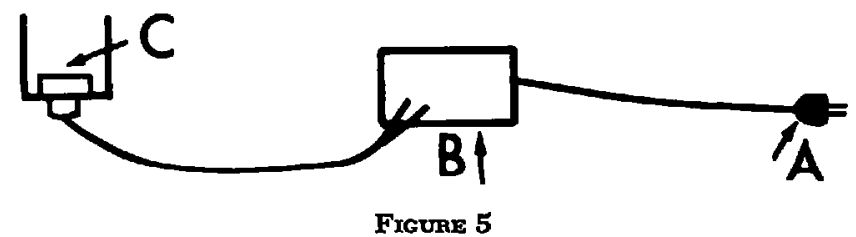

This method of nebulization produces large volumes of adequately nebulized liquids. The main disadvantage is that the equipment which is presently available on the market is expensive and requires technical supervision to provide proper function.

In view of the limitations of the preceding methods, a new concept for producing humidification was developed in the Inhalation Therapy Department of the Hospital for Sick Children in Toronto. This new concept is embodied in the WinLiz Nebulizer (Fig. 6). A gas is connected to the inlet port $A$ of the nebulizer and flows into the pressure chamber $B$. The gas passes through a fixed orifice, $C$, which is centred in the plate $D$. Liquid is allowed to fall upon the plate $D$, and as the gas passes through the orifice $C$ into the upper chamber it expands and picks up the liquid. The gas and liquid then strike the horseshoe-shaped bafle, $E$, and the liquid is broken into small particles.

The nebulizer has been subjected to laboratory investigations. Particle-size determinations were made with a six-stage cascade impactor. It is important to note that the viscosity of the solution is a vital factor in the analysis of particlesize determination. The findings are as follows:

Propylene glycol in distilled water (ratio 1:9)-0.5-1.75 microns (85\%)

Distilled water-0.5-2 microns (75\%)

$\mathrm{N}$-acetylcystine- $0.5-5$ microns (65\%). 


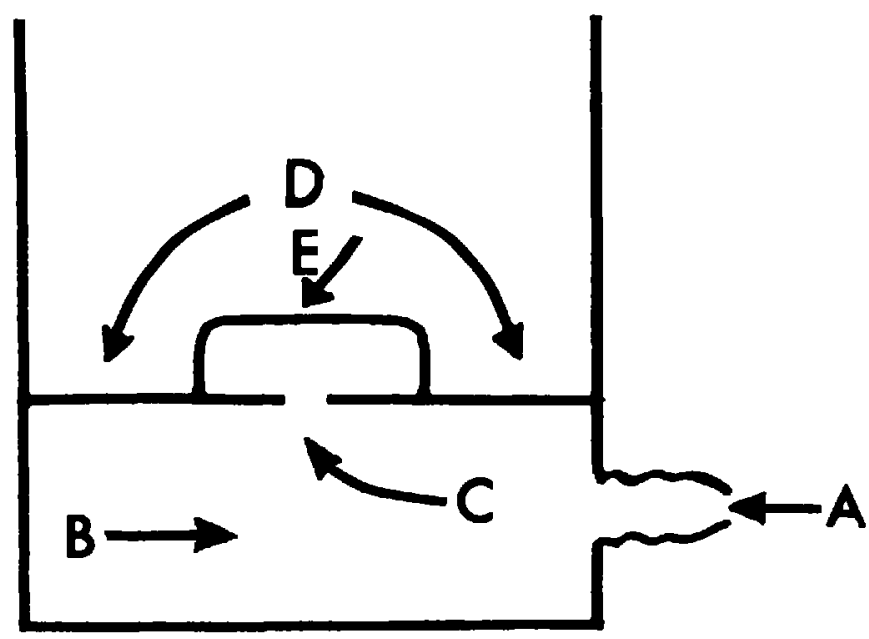

Figure 6

Particle-size distribution plots revealed that approximately 90 per cent (by weight) of the aerosol particles are smaller than 10 microns.

The WinLiz Nebulizer was compared with a leading conventional nebulizer, and the test showed that the former generated an aerosol which was 65 per cent more concentrated than that obtained with the conventional model.

The new nebulizer has been produced in two sizes-a large-capacity model for use in tents and incubators, and a small-capacity model for use in producing the aerosolization of medicants. It will operate under gas-flow rates ranging from $4 \mathrm{~L}$. $/ \mathrm{min}$. at the standard flow-meter outlet pressure up to $21.5 \mathrm{~L} . / \mathrm{min}$. at 30 p.s.i.

The advantages of the WinLiz Nebulizer can be summed up as follows:

1. It produces a large volume of humidity.

2. It produces a more concentrated aerosol.

3. It does not clog, since the liquid does not come into contact with the orifice of the plate separating the two chambers.

4. There is very little change in the concentration of the material being atomized. In many other nebulizers only a small fraction of the total liquid atomized leaves the nebulizer directly, and most of the solvent (e.g. sterile distilled water) is lost by continuous refluxing.

5. Its versatility gives it a wide range of application in anaesthesia and inhalation therapy.

6. Owing to their simplicity of construction, nebulizers of both capacities can be marketed at a reasonable cost.

\section{SUMMARY}

This paper has outlined the various methods of producing humidification, including a new concept devised and developed at the Hospital for Sick Children, Toronto. 